> La découverte récente de la voie Patched/Sonic Hedgehog et de son rôle dans la prédisposition génétique et dans l'oncogenèse du carcinome basocellulaire ont permis des avancées majeures dans la compréhension de la genèse de cette tumeur. Pour la première fois, des modèles murins de carcinome basocellulaire ont été développés, qui devraient permettre, d'une part, de mieux comprendre les autres mécanismes moléculaires impliqués et, d'autre part, de tester de nouvelles stratégies thérapeutiques préventives ou curatives, pour tenter de maîtriser les formes multiples de carcinome basocellulaire, souvent invalidantes. <

\section{Voie \\ Patched/Sonic \\ Hedgehog \\ et carcinomes \\ basocellulaires}

Nicole Basset-Seguin, Nadem Soufir

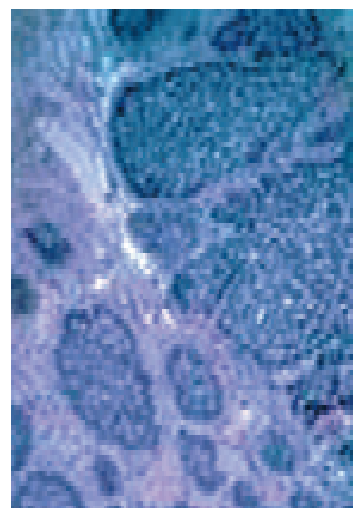

Anomalies génétiques et $\mathrm{CBC}$ avant la découverte

Les carcinomes cutanés sont les cancers les plus fréquents chez les sujets caucasiens ( $30 \%$ des cancers de l'adulte), deux tiers environ étant des carcinomes basocellulaires $(C B C)$. Ces derniers ont essentiellement un pouvoir invasif local et métastasent rarement. Le facteur carcinogène majeur est l'exposition solaire, ce qui est bien illustré par leur relative photodistribution. Des études épidémiologiques ont montré que le lien entre CBC et soleil était fortement lié à l'exposition UV intermittente dans l'enfance, à l'instar du mélanome [1], et beaucoup moins à l'exposition solaire cumulée au cours de la vie. L'incidence des CBC, estimée à 50000 nouveaux cas chaque année en France, augmente avec le degré d'ensoleillement et la latitude, avec un maximum en Australie, où les taux peuvent atteindre 788/100 000 habitants. La plupart des CBC sont sporadiques, mais de rares individus ont une maladie héréditaire prédisposant à leur survenue, le syndrome des hamartomes basocellulaires, ou syndrome de Gorlin. Depuis 1996, I'identification du gène responsable de cette génodermatose, le gène supresseur de tumeur patched (ptc) situé en 9q22, a permis de faire un grand pas dans la physiopathologie de cette tumeur $[2,3]$.

\section{Anomalies cytogénétiques}

Le CBC est caractérisé par un faible nombre d'anomalies chromosomiques. En effet, l'essentiel (75\%) des anomalies est retrouvé en $9 q 22$, zone de localisation du gène ptc [4] et, à un degré moindre, en 9p et lq. Quelques études plus récentes sont venues nuancer cette notion, d'autres anomalies cytogénétiques ayant été identifiées en $6 q, 4 q, 13 q$, $8 q, 7$ et $X$ dans les tumeurs les plus agressives [5]. Ainsi, outre le gène $p t c$, d'autres gènes suppresseurs de tumeurs pourraient jouer un rôle dans la physiopathologie du CBC.

\section{Gène $p 53$ et $C B C$}

C'est le gène le plus fréquemment muté dans les cancers, en général, et dans les CBC, en particulier (plus de $50 \%$ des cas) [6]. Les mutations observées sont localisées dans les exons 5 à 8 codant pour le domaine central de la protéine impliqué dans la liaison à l'ADN. II existe plusieurs points chauds mutationnels (codons 177, 179, 196 et 278) spécifiques des cancers cutanés. De plus, le spectre de mutation est différent selon la localisation de ces cancers : dans les CBC situés en peau photo-exposée, les 
mutations sont, dans plus de $80 \%$ des cas, de type UVBinduit (transitions $\mathrm{C} \rightarrow$ T ou mutations en tandem $\mathrm{CC} \rightarrow \pi$ à des sites dypirimidiques), alors que, dans les zones non photo-exposées, on observe plus souvent des transversions, suggérant d'autres facteurs que les UVB comme agents carcinogènes [7].

\section{Voie Patched/Sonic Hedgehog et oncogenèse des carcinomes basocellulaires}

Le gène $p t c$, dont les mutations sont à l'origine du syndrome de Gorlin, code pour la protéine Patched appartenant à la voie Sonic Hedgehog (Figure 1). De mieux en mieux caractérisée, cette voie de signalisation, impliquée dans le contrôle du développement embryonnaire et de la prolifération cellulaire, apparaît également capitale dans l'oncogenèse des $C B C$.

\section{La voie Patched/Sonic Hedgehog}

La protéine Patched, codée par le gène ptc, est un récepteur membranaire composé de douze segments transmembranaires et de deux grands segments extracellulaires requis pour la liaison du ligand Sonic Hedgehog [8]. Au moins deux homologues de Patched ont été décrits: Patched 2 [9] et TCR8, identifié dans les cancers du rein [10]. La protéine Sonic Hedgehog, codée par le gène shh localisé en $7 q 36$, est une protéine secrétée de $45 \mathrm{kDa}$, clivée en deux domaines: un domaine aminoterminal de $20 \mathrm{kDa}$ ayant une activité $\mathrm{Zn}$ hydrolase, et un domaine carboxyterminal de $25 \mathrm{kDa}$, autocatalytique, doué d'une activité cholestérol transférase. Le troisième acteur de la voie Sonic Hedgehog est Smoothened (Smo), codée par le gène Smoothened localisé en 7q31-32. II s'agit d'une protéine de 787 acides aminés appartenant à la famille des serpentines, récepteurs couplés à des protéines $G$, et qui présente une homologie avec la famille des récepteurs WNT [11]. Elle comprend un domaine aminoterminal et deux domaines ( $3^{e}$ boucle intracellulaire et $7^{e}$ domaine transmembranaire) impliqués dans la signalisation.

Activateur de la voie Sonic Hedgehog, Smo se comporte comme un protooncogène. Plusieurs mécanismes ont été proposés pour expliquer l'activation de Smo et la transduction du signal : changement conformationnel [13] et, plus récemment, régulation non stœchiométrique par Patched [14]. Patched présente en effet une homologie avec les transporteurs bactériens de protons, et la mutation, au cours du syndrome de Gorlin, de résidus conservés que l'on sait requis pour l'activité de ces transporteurs inhibe la fonction de Patched. La fonction suppresseur de tumeur de Patched sur Smo pourrait donc être indirecte, et il a été suggéré qu'elle passerait par une modification de la concentration d'une petite molécule [14].

\section{Mutations du gène ptc et syndrome de Gorlin}

Le syndrome de Gorlin est une affection rare, dont la prévalence est de 1/57 000 individus [15]. De transmission autosomique dominante, cette maladie est caractérisée par l'apparition de multiples CBC (avec un âge moyen de survenue de 20 ans), mais également d'autres tumeurs : médulloblastomes, fibromes ovariens et, plus rarement,

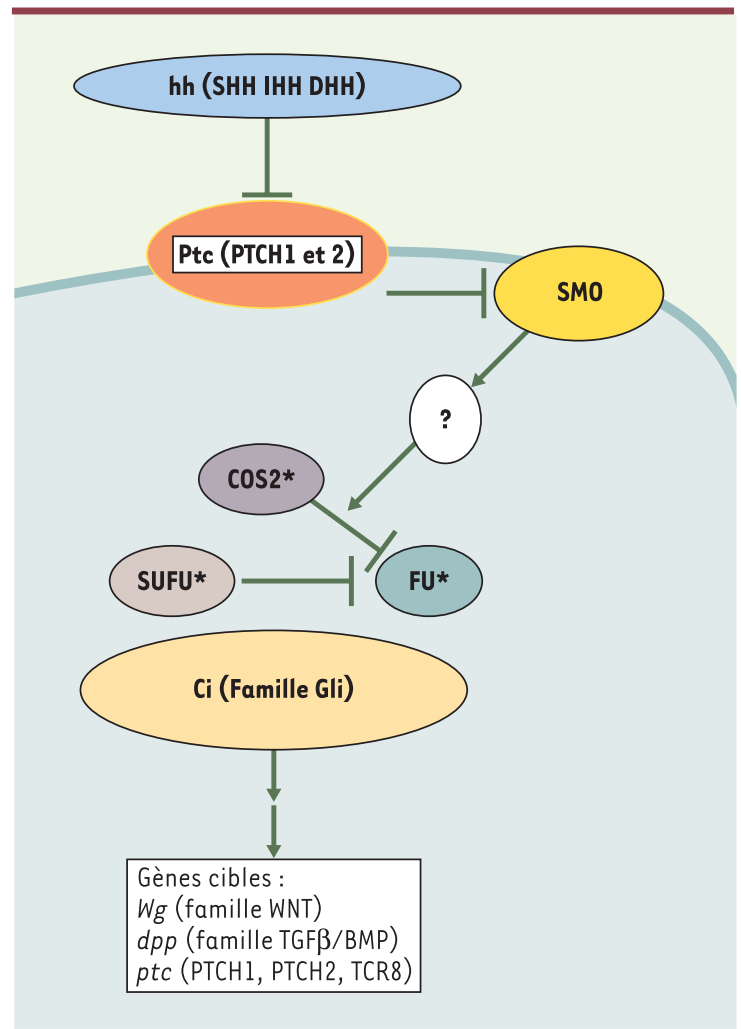

Figure 1. Voie Patched/Sonic Hedgehog. La liaison de Hedgehog (SHH, IHH ou DHH) sur Patched (PTCH) s'accompagne d'une endocytose des complexes Hedgehog/Patched avec, en corollaire, une diminution de la concentration de Patched dans la cellule, probablement via sa dégradation lysosomiale [1]. En l'absence de Hedgehog, Smo est inactif. La liaison de Hedgehog à Patched permet l'activation de Smo et la transduction du signal vers le noyau. Si la voie précise de transduction du signal via Smo n'est pas connue, la cible finale est représentée par l'activation du facteur de transcription Gli, homologue de Cubitus interruptus ( $\mathrm{Ci}$ ) chez la drosophile. Dans ce modèle, Ci forme, en l'absence de Hedgehog, un complexe tétramérique avec d'autres protéines comme Costal 2 (COS2), Fused (FU) et le facteur suppresseur de fused (SUFU). Ci est alors clivé en un fragment aminoterminal qui peut réprimer les gènes cibles dans le noyau. En présence du ligand Hedgehog, le complexe se dissocie et $\mathrm{Ci}$ entier joue son rôle d'activateur transcriptionnel. Chez les vertébrés, la réponse transcriptionnelle à Sonic Hedgehog est assurée par trois homologues de Ci, Glil et Gli2 (activateurs transcriptionnels), et Gli3 (répresseur transcriptionnel), qui agissent de concert afin de moduler l'expression des gènes cibles $W g$, codant pour les membres de la famille WNT, $d p p$, codant pour les bone morphogenic protein (BMP) et ptc, codant pour Patched. HH : Hedgehog ; SHH : Sonic Hedgehog; DHH : Desert Hedgehog ; IHH : Indian Hedgehog ; Fu : Fused ; SUFU: suppresseur de Fused ; * démontré chez la drosophile (d'après [8]). 
fibrosarcomes, méningiomes, rhabdomyosarcomes et fibromes cardiaques. Une caractéristique de ce syndrome est l'association avec différentes malformations [16] : kystes maxillaires (74\%), pits palmo-plantaires (87\%), macrocéphalie (50\%), hypertélorisme (42\%), bosse frontale (27\%), élargissement du visage ( $54 \%$ ), calcification de la faux du cerveau (65\%), côte bifide (26\%), anomalies vertébrales (25\%).

L'identification de mutations du gène ptc chez des individus atteints du syndrome de Gorlin [3] a permis de mieux comprendre l'observation des différentes anomalies, étant donné les fonctions de Patched dans le développement. La majorité des mutations sur le gène ptc sont des mutations stop aboutissant à une protéine tronquée $[2,3,17]$. Elles concernent la quasi-totalité du gène, sans zone connue de point chaud. Par ailleurs, des individus porteurs de la même mutation peuvent avoir des tableaux cliniques très différents : cette absence de corrélation génotype/phénotype suggère que d'autres facteurs, génétiques ou environnementaux, interviennent dans le développement de la maladie.

\section{Mutations des facteurs de la voie Sonic Hedgehog et $\mathrm{CBC}$ sporadiques}

Des mutations inhibitrices de ptc sont retrouvées dans $30 \%$ à $50 \%$ des CBC sporadiques. II s'agit dans plus de $50 \%$ des cas de mutations de type UVB-induit (transitions C $\rightarrow$ T et CC $\rightarrow$ T) [18] et, dans $70 \%$ cas, de mutations stop ou frameshift entraînant la synthèse d'une protéine tronquée [19]. Ces mutations lèvent l'inhibition de Patched sur Smoothened, entraînant ainsi l'activation permanente de la voie Sonic Hedgehog. Elles sont très souvent associées à une perte d'hétérozygotie en 9q22 (localisation du gène $p t c$ ) liée à une délétion du second allèle [20]. Ces données ont permis de confirmer l'importance de patched comme gène suppresseur de tumeur et de montrer que le développement du CBC obéit à la théorie de Knudson, l'inactivation du gène ptc nécessitant la survenue d'une mutation sur ses deux allèles [21].

Des mutations faux-sens, activatrices, du gène Smo ont été caractérisées dans $6 \%$ à $20 \%$ des CBC. Une transformation de fibroblastes primaires peut être obtenue par la cotransfection de la protéine $\varepsilon 1 A$ des adénovirus et de l'ADNc de deux mutants distincts de Smo (une mutation dans le $7^{\mathrm{e}}$ domaine transmembranaire, l'autre dans la partie carboxyterminale de la protéine), identifiés dans des CBC. Ces mutants, insensibles à l'inhibition de Patched, activent la voie Sonic Hedgehog [22]. Concernant la protéine Sonic Hedgehog, une seule mutation, activatrice, a été détectée dans une série de 43 cas de CBC sporadiques humains [23]; elle n'a pas été retrouvée ultérieurement [24].

\section{Autres facteurs de la voie de signalisation Sonic Hedgehog et $\mathrm{CBC}$}

\section{Récepteur du PDFG $\alpha$ (platelet-derived growth factor $\alpha$ )}

Le PDGFR $\alpha$ semble jouer un rôle important dans la formation de CBC induite par l'activation de la voie Sonic Hedgehog. En effet,
I'induction de PDGFR $\alpha$, l'un des gènes cibles de Glil, s'accompagne d'une activation de la voie de signalisation ras-ERK impliquée dans la prolifération cellulaire. Par ailleurs, l'expression de PDGFR $\alpha$ est élevée dans les CBC murins et humains et peut être diminuée par la réexpression de patched [25].

\section{Protéine FOXM1}

Les protéines de types FOX, composant une grande famille de facteurs de transcription, sont caractérisées par la présence d'un motif hélicoïdal conservé de 110 acides aminés englobant le domaine de liaison à l'ADN. Elles sont impliquées dans le développement embryonnaire et dans l'homéostasie des tissus adultes, jouant un rôle important dans la régulation de gènes impliqués dans la prolifération cellulaire, la différenciation et la transformation [26]. Certaines d'entre elles sont des effecteurs de la voie Sonic Hedgehog, notamment l'isoforme FOXMIb, spécifiquement induite dans les CBC par Glil : FOXMIb est donc l'un des gènes cibles de Glil, et la régulation de sa transcription pourrait constituer un mécanisme par lequel l'activation de la voie Sonic Hedgehog exerce ses effets mitogènes sur les kératinocytes [27].

\section{Modèles murins de CBC}

La découverte du rôle de la voie de signalisation Sonic Hedgehog dans la survenue des CBC a entraîné la mise au point de plusieurs modèles murins, qui ont permis de souligner l'importance de cette voie dans la physiopathologie de ces tumeurs.

\section{Souris transgéniques Sonic Hedgehog}

Les souris transgéniques surexprimant dans l'épiderme Sonic Hedgehog sous le contrôle du promoteur de la kératine 14 développent des anomalies du développement proches de celles observées au cours du syndrome de Gorlin, ainsi que des tumeurs CBC-like [23]. De même, la greffe, chez des souris nude, de kératinocytes humains exprimant constitutivement le facteur Sonic Hedgehog entraîne l'apparition de tumeurs CBClike au niveau de la peau greffée [28].

\section{Souris hétérozygotes $p t c^{+/-}$}

Ces souris développent spontanément de nombreuses anomalies du développement et des tumeurs multiples (médulloblastomes, rhabdomyosarcomes) [29]. Plusieurs modèles $p t c^{+/-}$, porteurs de délétions dans divers exons du gène, ont été développés, qui entraînent des phénotypes différents, liés au type de délétions comme au fond génétique. Sur le plan cutané, on retrouve des tumeurs folliculaires microscopiques proches des trichoblastomes humains, mais pas de CBC. Cependant, après irradiation par des rayonnements UV ou des radiations ionisantes, ces tumeurs se multiplient, grandissent et changent d'aspect histologique, ressemblant alors aux CBC humains, confirmant expérimentalement l'hypersensibilité aux UV et aux 
radiations des malades atteints de syndrome de Gorlin. Ce switch tumoral s'accompagne souvent de la perte de l'allèle restant de ptc et de mutations de $p 53$ dans les tumeurs [30]. Ces souris développent également des carcinomes épidermoïdes et des fibrosarcomes, dans lesquels il n'y a toutefois pas de perte d'expression de l'allèle sauvage de $p t c$, contrairement à ce qui est observé dans les CBC. Cependant, l'inactivation du second allèle de ptc est inconstante dans les CBC, la perte d'un seul allèle (haplo-insuffisance) donnant un avantage sélectif de croissance aux cellules.

\section{Souris transgéniques K5-Gli2 et K5-Gli1}

Des souris transgéniques surexprimant Gli2 sous le contrôle du promoteur de la kératine 5 développent des CBC multiples [31], suggérant que Gli2 est un effecteur important de la voie Sonic Hedgehog au cours des CBC. L'hyperexpression de Glil dans le même modèle entraîne, quant à elle, un phénotype différent, caractérisé par l'apparition de multiples tumeurs cutanées, principalement des trichoépithéliomes, mais d'une minorité seulement de CBC [32]. En fait, Gli2 possède un domaine fonctionnel aminoterminal ayant un effet répresseur sur l'expression de certains gènes, absent dans Glil [33]; ce domaine serait responsable des différences phénotypiques observées entre les souris transgéniques Glil et Gli2.

\section{Un espoir de traitement médical des CBC?}

Le traitement des CBC le plus couramment utilisé et le plus sûr à l'heure actuelle est la chirurgie. Cependant, $20 \%$ à $30 \%$ des patients présentent des CBC multiples, et il serait très intéressant de disposer d'un traitement médical (préventif ou curatif) évitant des exérèses chirurgicales multiples, fondé sur l'utilisation d'inactivateurs de la voie Sonic Hedgehog.

Les rétinoïdes, inhibiteurs de la voie Sonic Hedgehog, sont depuis longtemps proposés comme traitement préventif chez les sujets à risque de carcinomes cutanés, mais cet effet n'est réel qu'à des doses peu tolérées en pratique ce qui en limite l'utilisation.

Le pouvoir tératogène (anomalies cérébrales du développement, cyclopie dans les cas les plus sévères) de la jervine et de son dérivé, la 11-désoxojervine (ou cyclopamine), ont été mis en évidence dans les années 1950 [34]. Ces effet sont dus à l'inhibition spécifique de la voie Sonic Hedgehog [35], comme cela a été suggéré par les similitudes entre les anomalies observées et les malformations observées dans l'holoprosencéphalie, maladie génétique associée à des mutations inactivatrices de Sonic Hedgehog. Le mécanisme d'action de la cyclopamine est encore incertain, mais elle agit probablement au début de l'activation de la voie, et impliquerait la protéine Smo indépendamment de Patched. Son action est en effet atténuée chez les mutants de smo activés de manière constitutionnelle [36]. En se fixant directement sur Smo [37], la cyclopamine pourrait modifier son état de phosphorylation, sa localisation cellulaire et, peut-être, sa conformation. La cyclopamine a montré des vertus thérapeu- tiques intéressantes dans le traitement du médulloblastome chez la souris [38], tandis que son action sur les CBC est encore en cours d'évaluation dans les modèles murins.

\section{Conclusions et perspectives}

Le carcinome basocellulaire est lié avant tout à une anomalie dans la voie de signalisation Patched/Sonic Hedgehog. Les relations entre l'altération de cette voie et le rôle des UV dans la genèse de ces tumeurs mériteraient encore d'être précisés. Les travaux de recherche en cours dans de nombreuses équipes nous permettront sans nul doute de mieux comprendre et de contrôler le développement de ces tumeurs. $\diamond$

\section{SUMMARY}

Patched/Sonic Hedgehog pathway and basal cell

carcinoma

The recent discovery of the role of the Patched Sonic Hedgehog pathway in the physiopathogeny of BCC (basocellular carcinoma) and Gorlin's syndrome has greatly improved our knowledge on the mechanism of development of these tumors. For the first time, murine models have been developed allowing to further understand other molecular events implicated in such tumors as well as providing in vivo models to search for new curative or preventive therapeutical strategies which would be helpful to control CBC multiple forms, that are often disabling. $\diamond$

\section{RÉFÉRENCES}

1. Rosso S, Zanetti R, Martinez C, et al. The multicentre south European study «Helios ». II. Different sun exposure patterns in the aetiology of basal cell and squamous cell carcinomas of the skin. BrJ Cancer $1996 ; 73: 1447-54$.

2. Hahn H, Wicking C, Zaphiropoulous PG, et al. Mutations of the human homolog of Drosophila patched in the nevoid basal cell carcinoma syndrome. Cell 1996 ; $85: 841-51$.

3. Johnson RL, Rothman AL, Xie J, et al. Human homolog of patched, a candidate gene for the basal cell nevus syndrome. Science 1996 ; 272 : 1668-71.

4. Quinn AG, Sikkink S, Rees J. Delineation of two distinct deleted regions on chromosome 9 in human non-melanoma skin cancers. Genes Chrom Cancer 1994 ; $11: 222-5$.

5. Jin Y, Martins C, Salemark L, et al. Nonrandom karyotypic features in basal cell carcinomas of the skin. Cancer Genet Cytogenet $2001 ; 131: 109-19$.

6. Basset-Seguin N, Moles JP, Mils V, et al. TP53 tumor suppressor gene and skin carcinogenesis. J Invest Dermatol 1994 ; 103 : S102-6.

7. Matsumura $Y$, Nishigori C, Yagi T, et al. Characterization of $p 53$ gene mutations in basal-cell carcinomas: Comparison between sun-exposed and less-exposed skin areas. Int J Cancer $1996 ; 65: 778-80$.

8. Bale AE, Yu KP. The Hedgehog pathway and basal cell carcinomas. Hum Mol Genet $2001 ; 10: 757-62$.

9. Smyth I, Narang MA, Evans T, et al. Isolation and characterization of human Patched 2 (PTCH2), a putative tumour suppressor gene, in basal cell carcinoma and medulloblastoma on chromosome lp32. Hum Mol Genet $1999 ; 8$ : 291-7.

10. Gemmill RM, West JD, Boldog F, et al. The hereditary renal cell carcinoma $3 ; 8$ translocation fuses FHIT to a Patched-related gene, TRC8. Proc Natl Acad Sci USA 1998 ; 95 : 9572-7.

11. Van den Heuvel M, Ingham PW. Smoothened encodes a receptor-like serpentine protein required for Hedgehog signalling. Nature 1996 ; 382 : 547-51.

12. Incardona JP, Lee JH, Robertson CP, et al. Receptor-mediated endocytosis of soluble and membrane-tethered Sonic Hedgehog by Patched-1. Proc Natl Acad Sci USA $2000 ; 97: 12044-9$. 
13. Denef N, Neubuser D, Perez L, Cohen SM. Hedgehog induces opposite changes in turnover and subcellular localization of Patched and Smoothened. Cell 2000 ; $102: 521-31$

14. Taipale J, Cooper MK, Maiti T, Beachy PA. Patched acts catalytically to suppress the activity of Smoothened. Nature $2002 ; 418: 892-7$.

15. Farndon PA, Del Mastro RG, Evans DG, Kilpatrick MW. Location of gene for Gorlin syndrome. Lancet $1992 ; 339: 581-2$.

16. Kimonis VE, Goldsetin AM, Pastakia B, et al. Clinical manifestation in 105 persons with nevoid basal cell carcinoma syndrome. Am J Med Genet 1997 ; 69 : 299-308.

17. Toftgard R. Hedgehog signalling in cancer. Cell Mol Life Sci 2000 ; 57 : 1720-31.

18. Gailani MR, Stahle-Backdahl M, Leffell DJ, et al. The role of the human homologue of Drosophila patched in sporadic basal cell carcinomas. Nat Genet 1996 ; $14: 78-81$.

19. Aszterbaum M, Rothman A, Johnson RL, et al. Identification of mutations in the human Patched gene in sporadic basal cell carcinomas and in patients with the basal cell nevus syndrome. J Invest Dermatol 1998 ; $110: 885-8$.

20. Unden AB, Holmberg $\varepsilon$, Lundh-Rozell $B$, et al. Mutations in the human homologue of Drosophila patched (PTCH) in basal cell carcinomas and the Gorlin syndrome: different in vivo mechanisms of PTCH inactivation. Cancer Res $1996 ; 56: 4562-5$.

21. Ling G, Ahmadian A, Persson A, et al. Patched and $p 53$ gene alterations in sporadic and hereditary basal cell cancer. Oncogene $2001 ; 20: 7770-8$.

22. Xie J, Murone M, Luoh SM, et al. Activating Smoothened mutations in sporadic basal-cell carcinoma. Nature $1998 ; 391 ; 90-2$

23. Oro AE, Higgins KM, Hu Z, et al. Basal cell carcinomas in mice overexpressing Sonic Hedgehog. Science $1997 ; 276: 817-21$.

24. Wicking $C$, Evans $T$, Henk $B$, et al. No evidence for the Hl33y mutation in Sonic Hedgehog in a collection of common tumour types. Oncogene $1998 ; 16: 1091-3$.

25. Xie J, Aszterbaum M, Zhang X, et al. A role of PDGFRo in basal cell carcinoma proliferation. Proc Natl Acad Sci USA 2001 ; 98 : 9255-9.

26. Kaufman $\varepsilon$, Knochel W. Five years on the wings of fork head. Mech Dev 2001 ; $57: 3-20$.

27. Teh MT, Wong ST, Neill GW, et al. FOXMl is a downstream target of Glil in basal cell carcinomas. Cancer Res $2002 ; 62: 4773-80$.
28. Fan $\mathrm{H}$, Oro AE, Scott MP, Khavari PA. Induction of basal cell carcinoma features in transgenic human skin expressing Sonic Hedgehog. Nat Med 1997 ; 3 : 788-92.

29. Hahn H, Wojnowski L, Zimmer AM, et al. Rhabdomyosarcomas and radiation hypersensitivity in a mouse model of Gorlin syndrome. Nat Med 1198 ; 4 : 619-22.

30. Aszterbaum M, Epstein J, Oro A, et al. Ultraviolet and ionizing radiation enhance the growth of BCCs and trichoblastomas in patched heterozygous knockout mice. Nat Med 1999; 5 : 1285-91.

31. Grachtchouk M, Mo R, Yu S, et al. Basal cell carcinomas in mice overexpressing Gli2 in skin. Nat Genet $2000 ; 24: 216-7$.

32. Nilsson $M$, Unden $A B$, Krause $D$, et al. Induction of basal cell carcinomas and trichoepitheliomas in mice overexpressing GLI-1. Proc Natl Acad Sci USA 2000 ; 97 : 3438-43.

33. Sasaki H, Nishizaki Y, Hui C, et al. Regulation of Gli2 and Gli3 activities by an amino-terminal repression domain: implication of Gli2 and Gli3 as primary mediators of Shh signaling. Development $1999 ; 126: 3915-24$

34. Keeler RF, Binns W. Teratogenic compounds of Veratrum californicum (Durand). V. Comparison of cyclopian effects of steroidal alkaloids from the plant and structurally related compounds from other sources. Teratology $1968 ; 1: 5-10$

35. Incardona JP, Gaffield W, Kapur RP, Roelink H. The teratogenic Veratrum alkaloid cyclopamine inhibits Sonic Hedgehog signal transduction. Development 1998 ; $125: 3553-62$.

36. Taipale J, Chen JK, Cooper MK, et al. Effects of oncogenic mutations in Smoothened and Patched can be reversed by cyclopamine. Nature 2000; $406: 1005-9$.

37. Chen JK, Taipale J, Cooper MK, Beachy PA. Inhibition of Hedgehog signaling by direct binding of cyclopamine to Smoothened. Genes Dev $2002 ; 16: 2743-8$

38. Berman DM, Karhadkar SS, Hallahan AR, et al. Medulloblastoma growth inhibition by Hedgehog pathway blockade. Science 2002 ; 297 : 1559-61.

\section{TIRÉS À PART}

N. Basset-Seguin

\section{Tarifs d'abonnement pour M/S - 2004}

\section{Canada $^{1} \quad$ USA/Mexique ${ }^{1}$ France $^{2} \quad$ UE et Suisse ${ }^{2}$ Autres pays $^{2}$}

\begin{tabular}{llll}
\hline Particuliers & $\square 98 \$$ CAN & $\square 98$ US \\
\hline Institutions & $\square 195 \$$ CAN & $\square 195 \$$ US
\end{tabular}$\quad \begin{gathered}\text { Pour la France et autres pays, } \\
\text { communiquer avec EDK }\end{gathered}$

sur présentation de photocopie $R^{\circ} / V^{\circ}$ de la carte d'étudiant

\section{MES COORDONNÉES}

NOM PRÉNOM

SPÉCIALITÉ

ADRESSE

VILLE CODE POSTAL

PAYS TEL

COURRIEL

\section{MON RÈGLEMENT}

$\square$ Par chèque à l'ordre de Médecine/Sciences (Canada, USA et Mexique)

$\square$ Par chèque à l'ordre de EDK (France, UE et Suisse)

$\square$ Par carte de crédit $\square$ Visa $\square$ Mastercard

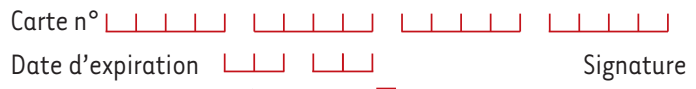

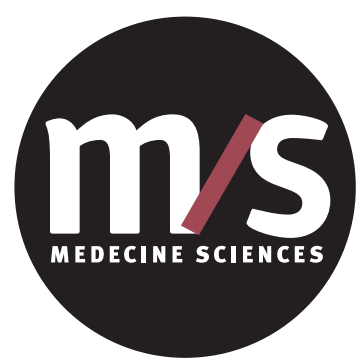

À découper et à renvoyer à :

${ }^{1}$ Médecine/Sciences

500 , rue Sherbrooke Ouest bureau 800

Montréal (Québec)

H3A 3C6 Canada medecine.sciences@ sympatico.ca

\section{${ }^{2} E D K$}

10 , villa d'Orléans

75014 Paris

France

edk@edk.fr

Conformément à la loi Informatique et Libertés du 6 janvier 1978, vous disposez d'un droit d'accès et de rectification des données personelles vous concernant. 